Please do not remove this page

RMIT

UNIVERSITY

\title{
Love mode SAW sensors with ZnO layer operating in gas and liquid media
}

Kalantar-Zadeh, Koroush; Wlodarski, W; Trinchi, Adrian; Holland, Anthony; Galatsis, Kosmas

https://researchrepository.rmit.edu.au/esploro/outputs/9921857973901341/filesAndLinks?institution=61RMIT_INST\&index=null

Kalantar-Zadeh, K., Wlodarski, W., Trinchi, A., Holland, A., \& Galatsis, K. (2002). Love mode SAW sensors with ZnO layer operating in gas and liquid media. Proceedings of the 2002 IEEE International Frequency Control Symposium and PDA Exhibition, 268-272. https://doi.org/10.1109/FREQ.2002.1075887

Published Version: https://doi.org/10.1109/FREQ.2002.1075887

Repository homepage: https://researchrepository.rmit.edu.au

(c) 2002 IEEE. Personal use of this material is permitted. However, permission to reprint/republish this material for advertising or promotional purposes or for creating new collective works for resale or redistribution to servers or lists, or to reuse any copyrighted component of this work in other works must be obtained from the IEEE.

Downloaded On 2023/04/26 23:35:49 +1000 


\title{
Love Mode SAW Sensors with ZnO layer Operating in Gas and Liquid Media
}

\author{
Kourosh Kalantar-Zadeh ${ }^{1,2}$, Wojtek Wlodarski ${ }^{1,2}$, Adrian Trinchi ${ }^{1,2}$, Anthony Holland ${ }^{1}$, \\ Kosmas Galatsis ${ }^{1,2}$ \\ 'RMIT University, School of Electrical and Computer Engineering, Melbourne, Australia \\ ${ }^{2} \mathrm{CRC}$ for Microtechnology, Melbourne, Australia
}

\begin{abstract}
Novel layered Surface Acoustic Wave (SAW) sensors, based on a $\mathrm{ZnO} / 90^{\circ}$ rotated ST-cut quartz crystal structure, were fabricated. They were employed for liquid and gas sensing applications. Their mass detection limit in liquid media is as low as $100 \mathrm{pg} / \mathrm{cm}^{2}$. Furthermore, these sensors are able to sense oxygen gas concentrations as low as $0.2 \mathrm{ppm}$ in nitrogen gas.
\end{abstract}

Keywords- Sensor, gas, immunosensor, SAW, layer, ZnO

\section{INTRODUCTION}

Surface Acoustic Wave (SAW) devices were first introduced by White and Voltmer [1] at the University of California, at Berkely. Their idea was ingenious but simple. They found out that by patterning interdigital transducers on piezoelectric substrates with the crystal cuts that allow the propagation of Rayleigh SAWs, the frequency spectrum of the device can be selectively tailored. The electronic industry quickly appreciated such an idea and applied SAW structures for the design and fabrications of analogue devices operating at selected frequencies [2],[3]. Passive SAW devices are now ubiquitous with applications ranging from professional radars and communications systems to consumer areas such as TV, pagers, mobile phones [4] and sensors [5].

Sensors that employ acoustic waves comprise a versatile class of structures [6] and are applied for both gas and liquid sensing applications. They are highly sensitive to the acoustic wave perturbation on the active area of the device. As the acoustic wave propagates through the bulk or on the surface of the device, any changes to the characteristic of the propagation path affect the velocity and amplitude of the wave [7]. The change in velocity can be monitored by measuring the frequency or phase characteristics of the sensor. These changes are correlated to the corresponding physical or chemical quantity that is being measured.

By depositing acoustic wave guiding layers onto the active area of SAW devices unique qualities can be obtained. Layered SAW devices generally have higher sensitivities comparing to that of blank SAW devices and the characteristics of SAWs can be controlled by changing the layered materials and thicknesses. In this paper, a layered SAW device with piezoelectric guiding layer and piezoelectric substrate [8],[9] for sensing applications will be introduced.

\section{LOVE MODE SENSORS}

Layered SAW devices are favourable due to their high sensitivity resulting from strong energy confinement in the guiding layers. Among them, Love mode devices have the most remarkable characteristic [10],[11],[12]. The movement of particles on the active sensing area is shear horizontal with a small normal component. As a result, the sensor can operate in both gas and liquid media.

To fabricate a Love mode device, the substrate cut should allow the propagation of Skimming Surface Bulk Wave (SSBW) or leaky SAW [2]. By depositing films on the substrate, in which the speed of shear horizontal acoustic wave is less than that of the substrate, SSBW or Leaky SAW is changed to Love wave.

In this research, novel Love mode SAW sensors, with $\mathrm{ZnO}$ layer as the wave-guiding films, were applied for sensing applications [9]. $\mathrm{ZnO}$ is a multi-functional material with high chemical stability [13]. It is widely applied as a gas sensitive material and as a piezoelectric, it has a high electromechanical coupling coefficient [14]. $90^{\circ}$ rotated ST-cut quartz crystal was selected as the substrate. It allows the propagation of SSBW.

In this research, a system incorporating Love mode SAW devices, was set up for conducting gas sensing and immunosensing experiments.

For gas sensing applications, the $\mathrm{ZnO}$ layer performs as both the acoustic wave guiding media and the gas sensitive film. For immunosensing applications, a thin gold layer was deposited onto the active surface of the device as the selective layer. Immobilization of Immunoglobulin $G$ (IgG) onto the gold surface was carried out to investigate the mass sensitivity of the sensors in the liquid media.

\section{THEORY}

When a Love mode SAW propagates in the layered media, the electric field associated with it can interact with the carriers that are adsorbed into the layer of the device. Therefore, it is possible that the Love mode SAW properties, such as the velocity and attenuation, will vary as film conductivity changes. The variation in operational frequency can be approximated by [15]:

$$
|\Delta f|=f \frac{K^{2}}{2} \frac{1}{1+\left(\frac{\sigma_{O R}}{\sigma_{o}}\right)^{2}},
$$


where $\sigma_{O R}=V_{0}\left(\varepsilon_{S}+\varepsilon_{A}\right)$ with $V_{0}$ as the speed of propagation of the acoustic waves in the substrate. $K^{2}$ is the electromechanical coupling coefficient, $f$ is the operational frequency and $\Delta f$ is the frequency shift that is caused by the perturbation of the electric field on the surface of the device. The term $\sigma_{\mathrm{OR}}$ is referred to as the resonant conductivity. $\varepsilon_{S}$ and $\varepsilon_{A}$ are the substrate and ambient atmosphere dielectric constant respectively, and $\sigma_{0}$ is the conductivity of the layer. $\sigma_{0}=\sigma . h$ where $\sigma$ is the bulk conductivity of the layer and $h$ is the layer thickness.

In immunosensing applications, the parameter of interest is mass sensitivity. Mass sensitivity is defined as the incremental signal change occurring in response to an incremental change in mass per unit area of the surface of the sensor. If the thickness of the film is less than one tenth of the acoustic wavelength, then first order perturbation theory can be used to calculate the change of the operational frequency [16]. Thus the change in frequency is given by:

$$
\Delta f=-\frac{V}{4} f \rho h\left[1-\left(\frac{V}{V_{S 2}}\right)^{2}\right]|\hat{v}|_{z=0}^{2}
$$

The substrate is considered semi-infinite with a layer of thickness of $h$ deposited onto the surface. In equation (2), $|\hat{v}|_{z=0}$ is the particle velocity at the surface of the layer, $\rho$ is the density and $V_{S 2}$ is the shear velocity of the deposited layer. The frequency shift $(\Delta f)$ depends on the acoustic phase velocity in the media $(V)$, the operational frequency $f_{0}$, $\rho h$ which represents the mass loading per unit area, $1-\left(\frac{V}{V_{s 2}}\right)^{2}$, which represents a reduction in mass sensitivity with the increase of the thickness of the layer, and on $|\hat{v}|_{z=0}$.

\section{FABRICATION OF THE DEVICE}

Depositing $\mathrm{ZnO}$ or $\mathrm{SiO}_{2}$ layers onto ST-cut quartz crystal substrate forms the required condition for Love mode wave propagation. The shear horizontal acoustic wave velocity in $\mathrm{ZnO}$ layer, $\mathrm{SiO}_{2}$ layer and $\mathrm{ST}$-cut quartz substrate are $2650 \mathrm{~m} / \mathrm{s}, 2850 \mathrm{~m} / \mathrm{s}$ and $5060 \mathrm{~m} / \mathrm{s}$, respectively.

$\mathrm{ZnO}$ films were deposited using r.f. magnetron sputterer [8]. These $\mathrm{ZnO}$ films have cylindrical grains grown on initial layer of randomly oriented crystallites. The radius of the $\mathrm{ZnO}$ grains varies between 50 to $200 \mathrm{~nm}$ (Fig. 1).

The IDTs (Inter-Digital Transducers) were patterned between the substrate and the $\mathrm{ZnO}$ layer. The IDTs electrode periodicity was $50 \mu \mathrm{m}$. The device for gas sensing application consists of 16 IDTs in the input port and 64 IDTs in the output port. The centre-to-centre IDTs distance was 60 wavelengths and aperture was $2.5 \mathrm{~mm}$. The device for immunosensing applicatons has 64 IDTs in input and output with centre-to-centre distance of 84 wavelengths.

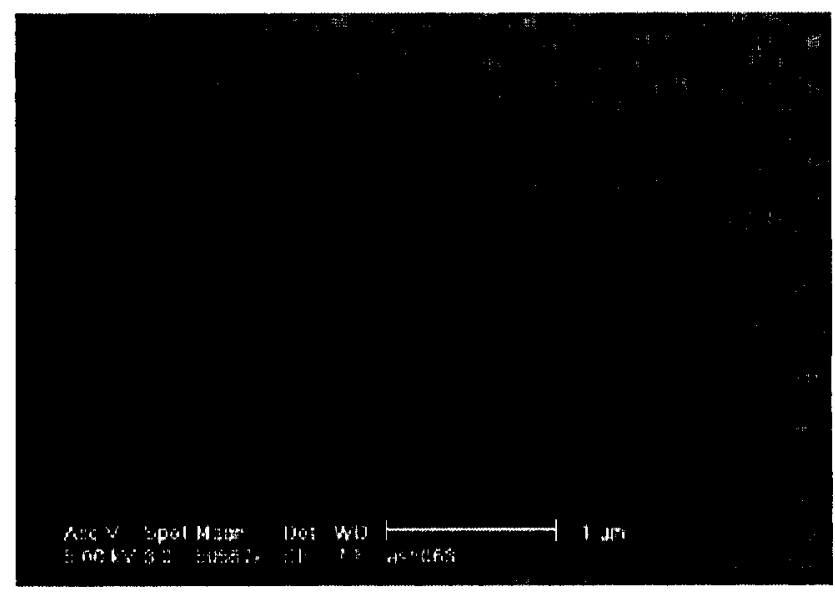

Fig. 1 SEM micrographs of the surface of the sputtered $\mathrm{ZnO}$.

X-Ray Diffraction (XRD) tests were used in the $2 \theta$ scan mode to determine the orientation of $\mathrm{ZnO}$ grains. Fig. 2 shows the typical XRD patterns from the $\mathrm{ZnO}$ thin films on the ST-cut quartz substrate deposited by the r.f. planar magnetron sputterer. The dashed line is the XRD measurement for a poor $c$-axis orientation $\mathrm{ZnO}$ film deposited at high deposition rate. The continuous line is the $\mathrm{XRD}$ using a deposition rate at $0.6 \mu \mathrm{m} / \mathrm{h}$.

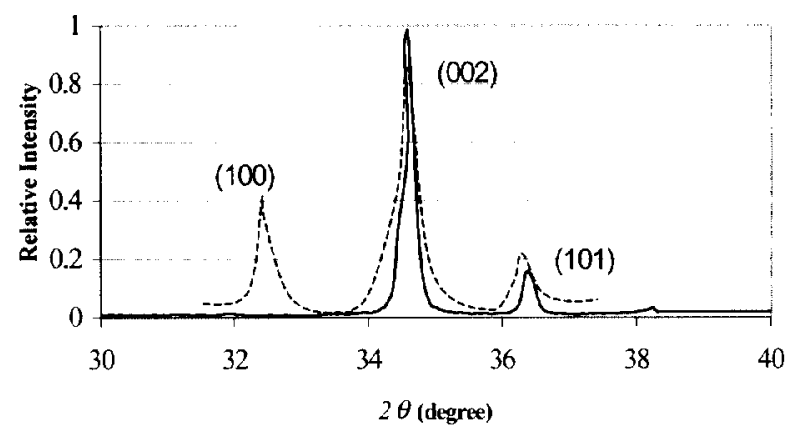

Fig. 2 XRD patterns from $\mathrm{ZnO}$ thin films on ST-cut quartz crystal wafers prepared by a planar r.f. magnetron sputterer: highly c-axis oriented is shown with the continuous line and poorly $\mathrm{c}$-axis oriented is shown with the dashed line.

With a planar r.f. magnetron the degree of orientation in the $c$-axis increases by using the optimum deposition parameters. The $(100)$ peak disappears and the (101) peak decreases significantly. Also the peak for $c$-axis (002) orientation became more dominant. It has been found that the degree of the $c$-axis orientation is strongly affected by employing different sputtering methods [17]. The $\mathrm{ZnO}$ thin 
films sputtered by the hemispherical system normally exhibit excellent $c$-axis orientation regardless of the substrate material [17].

The effect of $\mathrm{ZnO}$ annealing was investigated. When the films are annealed at $400^{\circ} \mathrm{C}$ and $500^{\circ} \mathrm{C}$, densification of the film occurred. Sintering (necking between the crystallites) was clearly observable in the film annealed at $500^{\circ} \mathrm{C}$. In addition, fracture section analysis by Scanning Electron Microscopy (SEM) revealed a significant shrinkage of the layer during the annealing step (a decrease in thickness from $4 \mu \mathrm{m}$ down to $2 \mu \mathrm{m}$ ). Annealing at $500^{\circ} \mathrm{C}$ also induces some significant structural changes. SEM analysis of the fractured surface of the film annealed at $500^{\circ}$ $\mathrm{C}$ shows the absence of columnar structure and the presence of a dense grainy structure. This micro-structural change is confirmed by the XRD analysis which reveal the absence of the peaks relative to the $<002>$ diffractions. Instead, the pattern exhibit only one peak at $26^{\circ}$ which could be attributed to the $<101>$ peak of quartz.

\section{THE SENSING SYSTEM}

The system is based on the oscillation circuit set-up [18]. The sensors delay line along with the amplifier make closed-loops electronic circuits that oscillate. The oscillation frequencies depend on the acoustic wave speed of propagation in the delay line. The system can be employed for gas sensing and liquid sensing application by replacing gas liquid delivery and liquid delivery systems in the set up.

\section{EXPERIMENTAL}

For gas sensing experiments, the thickness of the $\mathrm{ZnO}$ layer was $2.8 \mu \mathrm{m}$ giving an operational frequency of $90 \mathrm{MHz}$ for the periodicity of $50 \mu \mathrm{m}$. The sensor was heated to $350^{\circ} \mathrm{C}$ by a micro-heater located beneath the device.

The sensor was exposed to different concentrations of oxygen in nitrogen gas. The response to $100 \mathrm{ppm}$ of oxygen is shown in Fig. 3. Exposing the device to oxygen gas increases the operational frequency. This increase in frequency is almost $+18 \mathrm{kHz}$. For oxygen, the response and recovery times are continuous which stand for a single reaction on the surface.

The response of the sensor to different oxygen concentrations is shown in Fig. 4.

The response of the sensor to $50 \mathrm{ppm}$ is equal to $-11 \mathrm{kHz}$. If the response from this point onward would continue to be linear, then the response of the device to $0.5 \mathrm{ppm}$ will be equal to $110 \mathrm{~Hz}$. The frequency stability is better than $50 \mathrm{~Hz}$ in the gas media during the period of time of 5 hours.

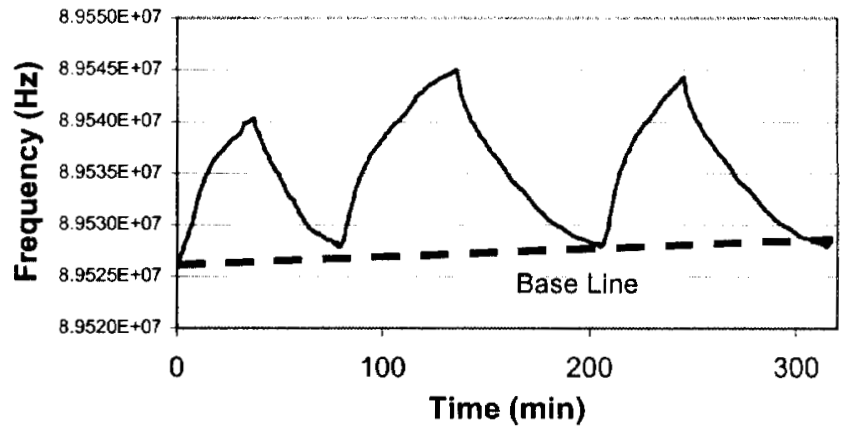

Fig. 3 Response of the Love mode device to a sequence of $100 \mathrm{ppm} \mathrm{O}_{2}$ in $\mathrm{N}_{2}$.

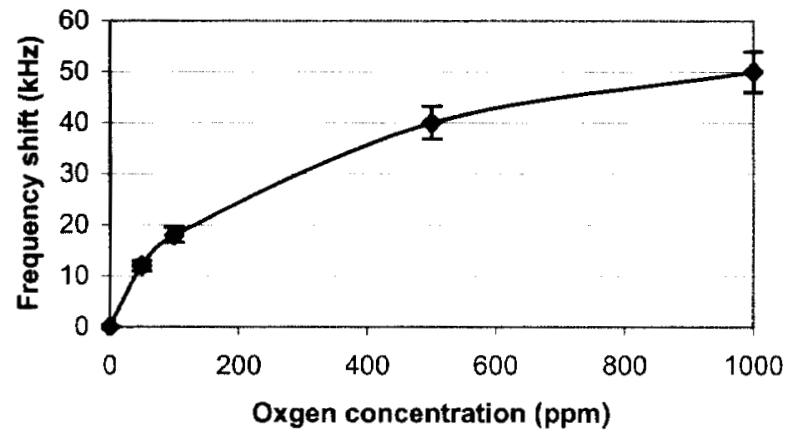

Fig. 4 Frequency shift of the Love mode $\mathrm{SAW}$ sensor with $\mathrm{ZnO}$ layer exposed to different oxygen concentrations.

The second series of experiments were conducted to examine the system's capability for immunosensing applications. A Love mode device with the $\mathrm{ZnO}$ layer of thickness $4 \mu \mathrm{m}$ was employed. For such a layer thickness, the sensitivity of the device is about $-800 \mathrm{~cm}^{2} / \mathrm{g}$ [9]. The gas delivery system was replaced by the liquid delivery system and dual delay line with a reference transducer was installed in the system. The resulting response was the frequency difference of the sensor and reference oscillator. The sensor then was exposed to IgG in PBS with different concentrations. Consequent pumping of IgG with two different concentrations is shown in Fig. 5. Consequent pumping of IgG with two different concentrations when after each exposure, surface of the sensor was washed with a mixture of alcohol in DI-water $(1: 10)$, is shown in Fig. 4. Firstly, $\mathrm{IgG}$ with the concentration of $1 \mu \mathrm{g} / \mathrm{mL}$ was pumped into the liquid cell. The flow rate was $50 \mu \mathrm{L} / \mathrm{min}$. The frequency shift was approximately $-20 \mathrm{kHz}$. Alcohol in DI-water (1:10) was then pumped into the cell to wash the gold surface. The washing time was $600 \mathrm{sec}$. Then the PBS buffer was pumped for $600 \mathrm{sec}$. The process was repeated, however this time a different concentration of IgG $(10 \mu \mathrm{g} / \mathrm{mL})$ was used. The frequency shift was $-100 \mathrm{kHz}$. 


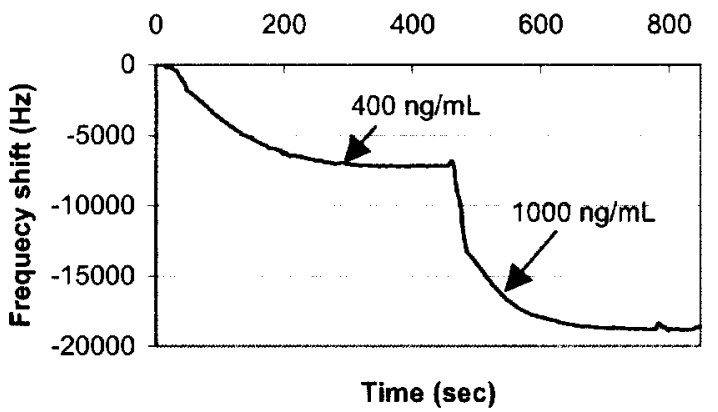

Fig. 5 Response of the Love mode SAW immunosensor to different concentrations of IgG after washing the active area of the sensor.

The frequency responses of the Love mode sensor versus different concentrations of IgG in PBS is shown in Fig. 6. In these measurements, the frequency shifts were measured after 500 seconds with the flow rate of $20 \mu \mathrm{L} / \mathrm{min}$.

The main advantage of the Love mode device with $\mathrm{ZnO}$ layer is the low detection limit which is practically achievable with this sensor. It shows a frequency shift of $-2.5 \mathrm{OHz}$ for $100 \mathrm{pg}$ of $\mathrm{IgG}$ in $1 \mathrm{~mL}$ PBS. The frequency change is better than $1 \mathrm{~Hz}$ in 30 minutes in static measurements.

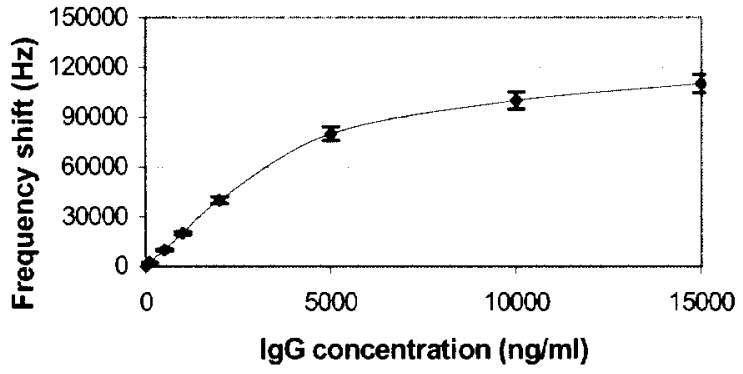

Fig. 6 IgG concentration versus frequency response of the Love mode sensor with $\mathrm{ZnO}$ layer. The frequency shift is the response of the device after $500 \mathrm{sec}$.

\section{CONCLUSIONS}

A novel Love mode SAW structure was fabricated and studied for gas and liquid sensing applications. The Love mode sensor structure was based on $\mathrm{ZnO} / 90^{\circ}$ rotated quartz crystal structure. The $\mathrm{ZnO}$ layer was deposited using r.f. magnetron sputterer. Due to the high electromechanical coupling coefficient of the structure, the device showed high conductometric sensitivity to different gas species and high mass sensitivity in response to IgG molecules. The sensor response was investigated for different concentrations of oxygen in nitrogen gas and different concentrations of IgG in PBS.

\section{ACKNOWLEDGMENT}

The authors would like to thank CRC for MicroTechnology, Australia, for the financial support.

REFERENCES

[1] R. M. White, F. W. Voltmer, "Direct Piezoelectric Coupling to Surface Elastic Waves," Appl. Phys. Lett., vol. 17, pp. 314-316, 1965.

[2] C. K. Campbell, Surface Acoustic Wave Devices for Mobile and Wireless Communications, Academic Press, USA, 1998.

[3] A. A. Oliner (ed.), Topics in Applied Physics: Acoustic, Surface Waves, Springer-Verlag, New York, 1978.

[4] D. P. Morgan, "History of SAW Devices," IEEE International Frequency Control Symposium, Los Angeles, California, USA, pp. 439-460, 1998,

[5] B. Drafts, "Acoustic Wave Technology," IEEE Trans. on Microwave Theory and Techniques, vol. 49, no. 4, pp. 795-802, 2001.

[6] J. W. Gray, S. J., Martin, R. M White, "Acoustic Wave Microsensors: Part I," Analytical Chemistry, vol. 65, no. 21, ,pp. 940-948, 1993.

[7] H. Wohltjen and R. Dessy, "Surface Acoustic Wave Probe for Chemical Analysis I: Introduction and Instrument Design," Anal. Chem., vol. 51, pp. 1458-1475, 1979.

[8] K. Kalantar-zadeh, W. Wlodarski, K. Galatsis, "Love Mode Layered Surface Acoustic Wave Filters," Electronics Letters, vol. 37, no. 12, pp. 802-803, 2001.

[9] K. Kalantar-Zadeh, A. Trinchi, W. Wlodarski, A. Holland, "A Novel Love Mode Device Based on A $\mathrm{ZnO} / \mathrm{ST}$-cut Quartz Crystal Structure For Sensing Applications," Sensors and Actuators $A$, in press.

[10] G.L. Harding, J. Du, P.R. Dencher, D. Barnett, and E. Howe, "Love Wave Acoustic Immunosensor Operating in Liquid," Sensors and Actuators A, vol. 61, pp. 279-286, 1997.

[11] B. Jakoby, M. J. Vellekoop, "Analysis and Optimization of Love Wave Liquid Sensors," IEEE Trans. Ultrasonics, Ferroelectr. Freq. Control, vol. 45, pp. 1293$1301,1998$.

[12] J. Du, G.L. Harding, J.A. Ogilvy, P.R. Dencher, and M. Lake, "A Study of Love-Wave Acoustic Sensors", Sensors and Actuators A, vol. 56, pp. 211-219, 1996.

[13] F. S. Hickernell, "Zinc Oxide Films for Acoustoelectric Device Applications," IEEE Transactions on Sonics and Ultrasonics, vol. SU-32, pp. 621-629, 1985.

[14] F.C.M. Van De Pol, "Thin-Film ZnO - Properties and Applications," Ceramic Bulletin, vol. 69, pp. 1959$1965,1990$.

[15] R. Lec, J. F. Vetelino, R. S. Falconer, and Z. Xu, "Macroscopic Theory of Surface Acoustic Wave Gas 
Microsensors," proceedings of 1988 IEEE Ultrasonics Symposium, pp. 585-589, 1988.

[16] Z. Wang and J. D. N. Cheeke, "Sensitivity Analysis for Love Mode Acoustic Gravimetric Sensors," Appl. Phys. Lett., vol. 64, no. 22, pp. 2940-2942, 1994.

[17] K. Wasa and S. Hayakawa, Handbook of Sputter Deposition Technology, Noyes Publications, Park Ridge, New Jersey, USA, 1995.

[18] U. Wolf, F. L. Dickert, G. K. Fischrauer, W. Greibl, and C. C. W. Ruppel, "SAW Sensors for Harsh Environments," IEEE Sensors Journal, vol. 1, pp. 4-13, 2001 . 